\title{
Systemtherapie des metastasierten Nierenzellkarzinoms
}

\author{
Luise Maute, Lothar Bergmann
}

\section{Was ist neu?}

- Erstlinientherapie: In der Erstlinientherapie des metastasierten Nierenzellkarzinoms stehen die Tyrosinkinasehemmer (TKI) Pazopanib und Sunitinib zur Verfügung. Für Patienten mit günstigem oder intermediärem Risikoprofil eignen sich IFNa / Bevacizumab sowie für Hochrisikopatienten der mTOR-Inhibitor Temsirolimus.

- Folgetherapie: In der Zweitlinientherapie nach Zytokintherapie können Axitinib oder Pazopanib und nach Vortherapie mit einer TKI/ VEGF gerichteten Therapie Axitinib oder Everolimus eingesetzt werden.

- Zweitlinie und weitere Therapieoptionen: In der Zweitlinie haben der PD1-Antikörper Nivolumab gegen Everolimus und Cabozantinib ebenfalls gegen Everolimus einen Überlebensvorteil gezeigt und sollten nach Zulassung in der Zweit- und Drittlinie primär eingesetzt werden.

\section{Aktueller Stand}

Jährlich erkranken in Deutschland etwa 16000 Menschen neu an einem Nierenzellkarzinom (RCC). Männer erkranken fast doppelt so häufig wie Frauen und das mittlere Erkrankungsalter liegt bei 60-70 Jahren [1, 2]. Die häufigste histologische Subentität ist mit einem Anteil von ca. $75 \%$ das klarzellige Nierenzellkarzinom. Die kürzlich erschienene neue WHO-Klassifikation unterscheidet neben dem klarzelligen RCC ca. 15 nicht-klarzellige Nierenzellkarzinome (ncRCC), wobei das papilläre RCC Typ I und II mit $15 \%$ und das chromophobe RCC mit ca. $4 \%$ die häufigsten Subentitäten sind [3]. Das klarzellige RCC geht meistens mit Mutationen des Hippel-vonLindau-Gens einher, beim papillären RCC Typ I sind c-Met-Mutationen beschrieben und auch die anderen Subtypen zeigen molekulare Charakteristika. Die Unterteilung der RCC wurde insbesondere anhand der Histologie und molekularen Charakteristika neu strukturiert ( Tab. 1).

Bei lokalisiertem RCC besteht ein kurativer Ansatz in einer Resektion des Primarius durch Nierenteilresektionen oder Nephrektomie. Die therapeutischen Optionen und die Prognose von Patienten mit metastasierter Erkrankung (25-30\% bereits bei Erstdiagnose) haben sich in den vergangenen Jahren durch die Zulassung neuer sogenannter „zielgerichteter“ Medikamente verbessert $[4,5]$. Bisher wurden sieben neue Substanzen zugelassen, die Tyrosinkinaseinhibitoren (TKI) Sunitinib, Sorafenib, Pazopanib und Axitinib, die mTOR-Inhibitoren Temsirolimus und Everolimus sowie der „vascular endothelial growth factor“ (VEGF) Antikörper Bevacizumab in Kombination mit IFN $\alpha$. Diese Substanzen führen zu einer Ver- längerung des progressionfreien Überlebens und einer medianen Lebenserwartung von über 2-3 Jahren [6-11]. Bei Vorhandensein von solitären oder wenigen Metastasen (synchron oder metachron) sollte immer die Resektabilität der Metastasen überprüft werden, insbesondere sofern keine neuen Metastasen in einer Verlaufsuntersuchung auftreten.

Eine systemische Therapie ist indiziert

- bei Irresektabilität oder klinisch nicht indizierter Resektion der Metastasen (z.B. keine R0Resektion wahrscheinlich, kurzfristig neu aufgetretene Metastasen) und Progredienz sowie

- bei tumorbedingter Symptomatik, die durch alleinige Lokaltherapie nicht behandelt werden kann.

\section{Erstlinientherapie}

In den meisten klinischen Studien zur Therapie des metastasierten RCC wurden ausschließlich oder zumindest weit überwiegend nur klarzellige RCCs als die häufigste Entität eingeschlossen [6-11]. In der kürzlich publizierten S3-Leitlinie Nierenzellkarzinome [5] sind das diagnostische Vorgehen, Therapien und Nachsorge detailliert Evidenz basiert erarbeitet. Die Prognose im metastasierten Stadium kann anhand von Scores eingeschätzt werden ( Tab. 2).

In der Erstlinientherapie stehen die Tyrosininhibitoren Sunitinib und Pazopanib zur Verfügung, die in der direkt vergleichenden Phase-III-Studie Comparz [12] eine ähnliche Effektivität hinsichtlich Ansprechen, progressionsfreiem Überleben und Gesamtüberleben zeigten. Dabei zeigte sich eine vergleichbare Wirksamkeit bezüglich des progressionsfreien Überlebens (Pazopanib: 8,4 Monate; Sunitinib: 9,5 Monate) und des Gesamtüberlebens (Pazopanib: 28,4 Monate; Sunitinib: 29,3 Monate; $p=0,275$ ) [12]. Das Toxizitätsprofil beider Substanzen ist etwas unterschiedlich und sollte bei der Wahl hinsichtlich bestehender Komorbiditäten beachtet werden.

Die Kombination von Interferon- $\alpha$ (IFN $\alpha)$ und dem VEGF-Inhibitor Bevacizumab sollte nur bei niedrigem oder intermediären Risikoprofil eingesetzt werden. Bei Patienten mit ungünstiger Prognose wurde kein Vorteil gegenüber IFN $\alpha$ alleine gezeigt [5, 8]. Im klinische Alltag wird diese Kombination nur noch selten eigesetzt. 


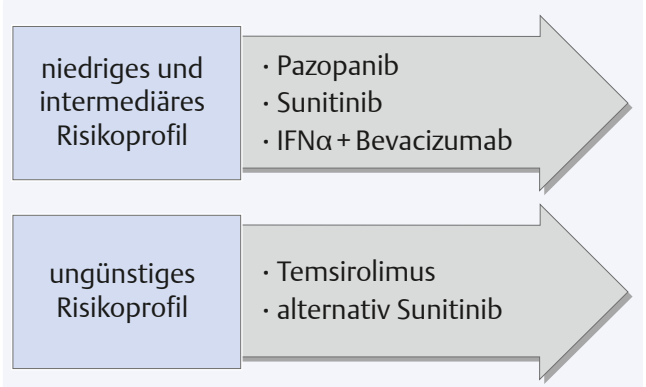

a

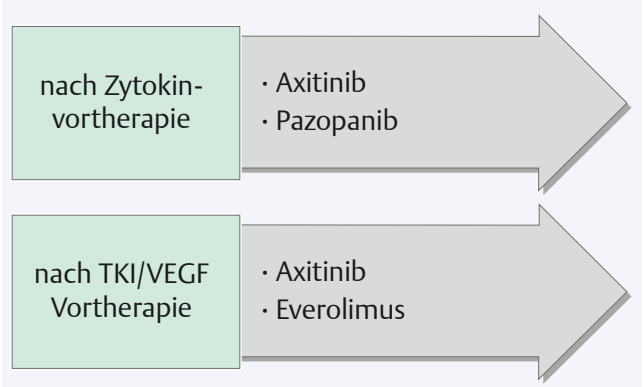

b

freie Überleben von 1,9 auf 4,0 Monate ( $p<0,0001$ ), wobei wegen des „crossover“ von ca. $70 \%$ der Patienten kein signifikanter Unterschied im Gesamtüberleben gezeigt werden konnte [15].

Zur Frage einer optimalen Therapiesequenz wurde die RECORD-3-Studie mit der Sequenz Everolimus dann Sunitinib vs. Sunitinib dann Everolimus in einer Nichtunterlegensheitsstudie geprüft [16]. Das mediane progressionsfreie Überleben war für Erstlinie Sunitinib gegenüber Everolimus mit 10,7 vs. 7,9 Monate höher. Das kombinierte progressionsfreie Überleben für Sunitinib/Everolimus war mit 25,8 gegenüber 21,1 Monate mit der Sequenz Everolimus / Sunitinib und das Gesamtüberleben mit 32,0 vs. 22,4 Monate (HR=1,24; Konfidenzintervall 0,94$1,64)$ verlängert. Damit sollte Everolimus nicht anstelle einer TKI in der Erstlinie appliziert werden $[5,16]$.

In der Switch-1-Studie wurde die Sequenz Sorafenib dann Sunitinib vs. Sunitinib dann Sorafenib geprüft [17]. Zwischen beiden Sequenzen zeigte sich kein signifikanter Unterschied im gesamten progressionsfreien Überleben.

\section{Zweitlinie und weitere Therapieoptionen}

In der Zweitlinie stehen aktuell vier Substanzen zur Verfügung, drei neue Substanzen mit anderen Wirkprinzipien sind zur Zulassung bei der European Medicine Agency (EMA) eingereicht (Cabozantinib, Nivolumab, Levantinib) und werden zukünftig die Sequenzabfolge verändern.

Axitinib wurde für die Zweitlinientherapie des mRCC nach Vorbehandlung mit Zytokinen oder Sunitinib zugelassen. Der TKI führte in der AXISStudie gegenüber Sorafenib zu einer Verlängerung des progressionsfreien Überlebens von im Median 6,7 vs. 4,7 Monaten ( $p<0,0001$ ) [14].

Nach Versagen einer TKI-Therapie kann der mTOR-Inhibitor Everolimus eingesetzt werden. Nach Sunitinib und/oder Sorafenib verlängerte Everolimus gegenüber Placebo das progressions-
Abb. 1 Therapiealgorithmus in $\operatorname{der}$ (a) Erstlinientherapie und (b) Zweitlinientherapie.
Eine sequenzielle Therapie sollte stets nach Versagen oder Unverträglichkeit einer vorangegan-

\footnotetext{
Nierenzelltumoren

- klarzelliges Nierenzellkarzinom

- multilokuläres zystisches Nierenzellkarzinom

- papilläres Nierenzellkarzinom

- hereditäre Leiomyomatosis und assoziiertes Nierenzellkarzinom (HLRCC)

- chromophobes Nierenzellkarzinom

- Ductus Bellini Karzinom

- medulläres Nierenzellkarzinom

- MiT familiäres Translokationskarzinom

- Succinat-Dehydrogenase-defizientes Nierenzellkarzinom

- muzinös tubuläres und Spindelzellkarzinom

- Nierenzellkarzinom bei erworbener zytischer Nierenerkrankung

- klarzellig papilläres Nierenzellkarzinom

- unklassifizierbares Nierenzallkarzinom

- papilläres Adenom

- Onkozytom
}

Tab. 1 Histologische Einteilung der Nierenzellkarzinome [3]. 


\section{MSKCC-Score}

- Karnofsky-Index<80\%

- Intervall zwischen Diagnose und Behandlungsbeginn < $1 \mathrm{Jahr}$

- korrigiertes Serum-Ca>10 mg/dl

- $\mathrm{Hb}<\mathrm{LLN}$

- $\mathrm{LDH}>1,5$ des Normwertes

\section{IMDC-Score}

- Karnofsky-Index<80\%

- Intervall zwischen Diagnose und Behandlungsbeginn < 1 Jahr

- korrigiertes Serum-Ca>10 mg/dl

- Neutrophilenzahl>Normwert

- Thrombozyten $>$ Normwert

Tab. 2 Prognostische Scores für metastasierte Nierenzellkarzinome [27, 28]. MSKCC = Memorial Sloan Kettering Cancer Center; IMDC = International Metastatic Renal Cell Carcinoma Database Consortium

genen Therapie angestrebt werden. Eine spezifische Sequenz von Substanzen kann derzeit allerdings nicht empfohlen werden ( $\bullet$ Abb. 1) [5].

\section{Klinische Relevanz \\ Für die Zweitlinientherapie des mRCC sind 4 Substanzen zugelassen: Axitinib, Pazopanib, Everolimus und Sorafinib. Everolimus sollte nicht in der Erstlinie anstelle eines Tyrosin- kinaseinhibitors eingesetzt werden, sondern erst, wenn die Therapie mit einem TKI versagt hat. Eine sequenzielle Therapie sollte stets nach Versagen oder Unverträglichkeit einer vorangegangenen Therapie angestrebt werden. Eine spezifische Sequenz von Substanzen kann derzeit nicht empfohlen werden.}

\section{Neue Therapieoptionen}

Die im folgenden Abschnitt erwähnten Substanzen sind derzeit noch nicht zugelassen.

In einer randomisierten Phase-II-Studie wurde der TKI Lenvatinib in einer Kombination mit Everolimus vs. Everolimus Mono und vs. Lenvatinib Mono beim metastastasierten RCC nach einer VEGF-gerichteten Therapie geprüft [18]. Durch die Kombination von Lenvatinib mit Everolimus konnte eine signifikante Verlängerung des progressionsfreien Überlebens von 14,6 vs. 5,5 Monate mit Everolimus allein $(p=0,0005)$ erreicht werden. Gegenüber Lenvatinib war der Unterschied mit 7,4 Mo-

Abb. 2 Zukünftiger Therapiealgorithmus nach Zulassung von Nivolumab und Cabozantinib mit Überlebensvorteil. nate nicht signifikant unterschiedlich. Allerdings war die Kombination mit einem relativ hohen Anteil an Grad 3 und 4 Toxizität assoziiert (71\%).

Cabozantinib, ein weiterer TKI, der außer VEGFR auch c-MET und AXL inhibiert, wurde in der Zweitlinie gegen Everolimus geprüft [19]. Das mediane progressionsfreie Überleben betrug 7,4 Monate mit Cabozantinib vs. 3,8 Monate mit Everolimus ( $\mathrm{p}<0,0001)$. Das Gesamtansprechen $21 \%$ vs. $5 \%$ mit Everolimus ( $p<0,0001)$ und insbesondere das Gesamtüberleben war signifikant erhöht. Allerdings wurde bei $60 \%$ der Patienten unter Cabozantinib die Dosis aufgrund von Toxizitäten reduziert, im Everolimus-Arm nur bei $25 \%$ [19].

Große Hoffnungen werden auf den Einsatz von „immune checkpoint“-Inhibitoren gesetzt. In einer Phase-III-Studie wurde der PD1 (programmed cell death) Antikörper Nivolumab gegen Everolimus als Vergleichsarm geprüft [20]. Das Gesamtüberleben war mit 25,0 vs. 19,6 Monate signifikant verlängert $(p=0,002)$. Das Gesamtansprechen war höher mit Nivolumab 25 vs. 5\% ( $<<0,001)$ während das progressionsfreie Überleben mit 4,6 vs. 4,4 Monate nicht unterschiedlich war. Grad 3 und 4 Toxizitäten traten in $19 \%$ unter Nivolumab und in $37 \%$ der Patienten unter Everolimus auf. Ein besonderes Problem in der bildgebenden Beurteilung des Ansprechens nach RECIST ist das Auftreten von sogenannten Pseudoprogressionen als Folge einer lokalen Immunreaktion, so dass hier eine Therapie über den ersten Progress hinaus diskutiert werden muss [21].

Mit Zulassung von Cabozantinib und Nivolumab nach Versagen einer VEGFR-gerichteter Therapie, die beide einen Überlebensvorteil gezeigt haben, wird sich zukünftig der Therapiealgorithmus ändern [22]. Beide Substanzen werden aufgrund des Überlebensvorteils in die Zweit- und Drittlinie rücken und die anderen Substanzen in nachgeordnete Sequenzen schieben ( $\triangleright$ Abb. 2).

\section{Klinische Relevanz}

Nivolumab und Cabozantinib zeigen gegenüber Everolimus einen Überlebensvorteil. Nach Zulassung sollen sie in der Zweit- und Drittlinie primär eingesetzt werden.

Nicht-klarzellige Nierenzellkarzinome I Es gibt wenige fundierte Studiendaten zu metastasierten nicht-klarzelligen Nierenzellkarzinomen. In einer Metaanalyse [23] wurde gezeigt, dass metastasierte nicht-klarzellige RCC eine ungünstigere Prognose unter zielgerichteten Therapien als klarzellige RCC haben. In drei kleinen prospektiv randomisierten Studien wurde Sunitinib gegen einen mTOR-Inhibitor (Everolimus, Temsirolimus) geprüft [24-26]. In allen diesen Studien zeigte sich eine leichte Tendenz zugunsten von 
Sunitinib. Inwieweit unterschiedliche histologische Subtypen mehr von einem Tyrosinkinaseoder mTOR-Inhibitor profitieren, lässt sich bei den kleinen Fallzahlen nicht abschließend entscheiden.

\section{Klinische Relevanz}

Derzeit sollten die nicht-klarzelligen RCC analog den klarzelligen RCC therapiert werden. Als zukünftige Optionen werden auch hier „immune checkpoint“ Inhibitoren und c-Met-Inhibitoren beim papillären RCC Typ I untersucht.

\section{Literatur}

1 Kaatsch P, Spix C, Katalinic A et al. Krebs in Deutschland 2009/2010. In Berlin: Robert Koch-Institut; Gesellschaft der epidemiologischen Krebsregister in Deutschland e. V.; (2013)

2 Corgna E, Betti M, Gatta G et al. Renal cancer. Crit Rev Oncol Hematol 2007; 64: 247-262

3 Moch H, Humphrey PA, Ulbright TM, Reuter VE. WHO Classification of Tumours of the Urinary System and Male Genital Organs. Fourth edition. IARC. Lyon 2016

4 Miller K, Bergmann L, Gschwend J et al. Interdisziplinäre Empfehlungen zur Behandlung des metastasierten Nierenzellkarzinoms. Aktuelle Urol 2015: 46: $151-157$

5 S3 Leitlinie Diagnostik, Therapie und Nachsorge des Nierenzellkarzinoms. 2015; www.awmf.org/ uploads/tx_szleitlinien/043-0170I-I_S3 Nierenzellkarzinom_2015-09.pdf (Letzter Zugriff 9.3.2016)

6 Fisher RI, Rosenberg SA, Fyfe G. Longterm survival update for high-dose recombinant interleukin-2 in patients with renal cell carcinoma. Cancer J Sci Am 2000; 6: Suppl 1: S55-S57

7 Escudier B, Goupil MG, Massard C, Fizazi K. Sequential therapy in renal cell carcinoma. Cancer 2009; 115: 2321-2326

8 Escudier B, Pluzanska A, Koralewski P et al. Bevacizumab plus interferon alfa-2a for treatment of metastatic renal cell carcinoma: A randomised, double-blind phase III trial Lancet 2007; 370: 2103-2111

9 Motzer RJ, Hutson TE, Tomczak P et al. Overall survival and updated results for sunitinib compared with interferon alfa in patients with metastatic renal cell carcinoma. J Clin Oncol 2009; 27: 3584-3590

10 Sternberg CN, Davis ID, Mardiak J et al.Pazopanib in locally advanced or metastatic renal cell carcinoma: results of a randomized phase III trial. J Clin Oncol 2010; 28: 1061-1068

11 Hudes G, Carducci M, Tomczak P et al. Temsirolimus, interferon alfa, or both for advanced renal-cell carcinoma. N Engl J Med 2007; 356: 2271-2281

12 Motzer RJ, Hutson TE, Cella D et al. Pazopanib versus sunitinib in metastatic renal-cell carcinoma. N Engl J Med 2013; 369: 722-731

13 Gore ME, Szczylik C, Porta C et al. Final results from the large sunitinib global expanded-access trial in metastatic renal cell carcinoma. Br J Cancer 2015; 113: $12-19$

14 Rini BI, Escudier B, Tomczak P et al. Comparative effectiveness of axitinib versus sorafenib in advanced renal cell carcinoma (AXIS): a randomised phase 3 trial. Lancet 2011; 378: 1931-1939

15 Motzer RJ, Escudier B, Oudard S et al. Phase 3 trial of everolimus for metastatic renal cell carcinoma: final results and analysis of prognostic factors. Cancer 2010; 116: 4256-4265

16 Motzer RJ, Barrios CH, Kim TM et al. Phase II randomized trial comparing sequential first-line everolimus and second-line sunitinib versus first-line sunitinib and second-line everolimus in patients with metastatic renal cell carcinoma. J Clin Oncol 2014; 32: 2765-2772

17 Eichelberg C, Vervenne WL, De Santis M et al. SWITCH: A Randomised, Sequential, Open-label Study to Evaluate the Efficacy and Safety of Sorafenib-sunitinib Versus Sunitinib-sorafenib in the Treatment of Metastatic Renal Cell Cancer. Eur Urol 2015; 68: 837-847

18 Motzer RJ, Hutson TE, Glen H et al. Lenvatinib, everolimus, and the combination in patients with metastatic renal cell carcinoma: a randomised, phase 2, open-label, multicentre trial. Lancet Oncol 2015; 16: 1473-1482

19 Choueiri TK, Escudier B, Powles Tet al. Cabozantinib versus Everolimus in Advanced Renal-Cell Carcinoma. N Engl ] Med 2015; 373: 1814-1823

20 Motzer RJ, Escudier B, McDermott DF et al. Nivolumab versus Everolimus in Advanced Renal-Cell Carcinoma. N Engl J Med 2015; 373 : 1803-1813

21 Wolchok JD, Hoos A, O’Day S et al. Guidelines for the evaluation of immune therapy activity in solid tumors: immune-related response criteria. Clin Cancer Res 2009; 15: 7412-7420

22 Powles T, Staehler M, Ljungberg B et al. Updated EAU guidelines for clear cell renal cancer patients who fail VEGF targeted therapy. Eur Urol 2016; 69: 4-6

23 Vera-Badillo FE, Templeton AJ, Duran I et al. Systemic therapy for non-clear cell renal cell carcinomas: a systematic review and meta-analysis. Eur Urol 2015; 67: 740-749

24 Armstrong AJ, Halabi S, Eisen T et al. Everolimus versus sunitinib for patients with metastatic non-clear cell renal cell carcinoma (ASPEN): a multicentre, open-label, randomised phase 2 trial. Lancet Oncol 2016, epub ahead of print

25 Tannir NM, Jonasch E, Albiges L et al. Everolimus Versus Sunitinib Prospective Evaluation in Metastatic Non-Clear Cell Renal Cell Carcinoma (ESPN): A Randomized Multicenter Phase 2 Trial. Eur Urol. 2015, doi: 10.1016/j.eururo.2015.10.049

26 Bergmann L, Grünwald V, Maute L et al. A Prospective Randomized Phase-II Trial with Temsirolimus vs. Sunitinib in non-clear Renal Cell Carcinoma (C-II-006). ECCO 2015, \#2617

27 Motzer RJ, Bacik J, Schwartz LH et al. Prognostic factors for survival in previously treated patients with metastatic renal cell carcinoma. J Clin Oncol. $2004 ; 22: 454-463$

28 Ko JJ, Xie W, Kroeger $\mathrm{N}$ et al. The International Metastatic Renal Cell Carcinoma Database Consortium model as a prognostic tool in patients with metastatic renal cell carcinoma previously treated with first-line targeted therapy: a population-based study. Lancet Oncol 2015; 16 : 293-300

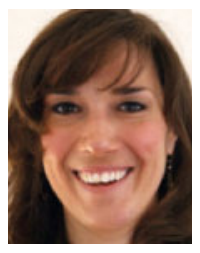

Dr. med. Luise Maute ist Fachärztin für Innere Medizin und Hämatologie / Onkologie an der Medizinischen Klinik II der Universitätsklinik Frankfurt. luise.maute@kgu.de

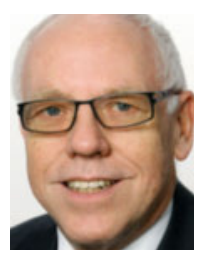

Prof. Dr.med. Lothar Bergmann ist Stellvertretender Direktor der Medizinischen Klinik II der Universitätsklinik Frankfurt.

l.bergmann@ em.uni-frankfurt.de

\section{Interessenkonflikt}

L.M. hat Honorare für Advisory Boards von Pfizer und BMS sowie für Workshops von Pfizer erhalten. L.B. hat an Advisory Boards von Pfizer, Novartis und BMS teilgenommen. Er erklärt, dass er keine persönlichen Honorare erhalten hat.

DOI 10.1055/s-0042-104594

Dtsch Med Wochenschr 2016; 141: 466-469 (c) Georg Thieme Verlag KG . Stuttgart · New York . ISSN 0012-0472 